\title{
LED 冷光源蓝光治疗病理性黄疸的临床评估
}

\section{Clinical Evaluation of LED Cold Light Source Blue Light in the Treatment of Pathological}

Jaundice

何文红 刘爱玲 麻文广 杨柳

Wenhong He Ailing Liu Wenguang Ma Liu Yang



【摘要】为了比较 LED 蓝光及普通蓝光治疗新生儿黄㾝的疗效,将 60 例新生儿黄㾝分 为 2 组, 分别用 LED 蓝光及普通蓝光治疗, 比较 2 组之间疗效及副作用是否有统计学意 义。结果显示, LED 蓝光治疗新生儿黄㾝疗效优于普通蓝光, 2 组间 LED 蓝光发热的副作用 明显小于普通蓝光, 皮疹、及腹泻发生率无明显差异。由此得出, LED 蓝光治疗黄㾝的效果 优于普通蓝光, 而副作用小于普通蓝光。

【Abstract】In order to compare the efficacy of LED blue light and common blue light in the treatment of neonatal jaundice, 60 cases of neonatal jaundice are divided into two groups and treated with LED blue light and ordinary blue light respectively. The curative effect and side effects between the two groups are compared. The results show that the curative effect of LED blue light in the treatment of neonatal jaundice is better than that of ordinary blue light. There is no significant difference in the side effect of LED blue light fever between the two groups compared with that of ordinary blue light, rash and diarrhea. It is concluded that the effect of LED blue light in the treatment of jaundice is better than that of ordinaryblue light, and the side effect is less than that of ordinary blue light.

【关键词】LED 蓝光; 新生儿黄㾝; 护理

【Keywords】LED blue light; neonataljaundice; nursing

【DOI】10.36012/pmr.v1i1.106

\section{1 研究内容}

\section{1 研究对象标准}

新生儿黄疸诊断均符合(实用新生儿学)第四版诊断标 准 ${ }^{[1]}$ 。

纳入标准: 黄疸出现的时间 2 5 天, 经皮胆红素测定符合 诊断新生儿黄㾝且达到光疗指证,排除标准: (1)ABO 及 $\mathrm{Rh}$ 溶
血病; (2)光疗前肝肾功能异常; 3)头皮血肿和颅内出血。

\section{2 研究对象与仪器}

选择 2018 年 1 月一-2018 年 12 月在南阳油田总医院儿科 接受蓝光治疗的新生儿黄疸 60 例, 分为 LED 蓝光组和普通蓝 光组, 2 组在性别、年龄、体重、皮肤黄染程度等情况无明显差 异, 具有可比性; 科室应用的光疗箱型号 BB-100G, KJ8000 经 皮黄疸仪。 
临床医学研究 Clinical Medicine Research

\section{2 结果}

与普通组比较, LED 组治疗后第 $24 、 48$ 小时经皮胆红素 值明显下降, 且差异有统计学意义 $(P<0.05)$, 详见表 1 。

表 1 光疗前后经皮测定胆红素值比较 ( $\mu \mathrm{mol} / \mathrm{L}$ )

\begin{tabular}{c|c|c|c|c}
\hline 组别 & 例数 & 治疗前 & 治疗后 24 小时 & 治疗后 48 小时 \\
\hline LED 组 & 30 & $219.3 \pm 10.3$ & $186.4 \pm 2.0$ & $109.4 \pm 3.9$ \\
\hline 普通组 & 30 & $230.5 \pm 5.1$ & $197.8 \pm 13.8$ & $135.1 \pm 8.9$ \\
\hline$P$ 值 & & $>0.05$ & $<0.05$ & $<0.05$ \\
\hline
\end{tabular}

注: $P$ 为 $\chi^{2}$ 检验中假设为真时的概率。

根据 $\chi^{2}$ 检验, 副作用发热的发生率 LED 蓝光组少于普通 蓝光组。2 者比较差异有统计学意义, 余副作用的发生率的差 异均无统计学意义。详见表 2 。

表 22 组光疗副作用的分布及发生率的比较

\begin{tabular}{c|c|c|c|c}
\hline 组别 & 发热 & 皮疹 & 腹泻 & 脱水 \\
\hline 普通组 & 11 & 3 & 4 & 2 \\
\hline LED 组 & 5 & 2 & 4 & 2 \\
\hline 2 组比较 & $P<0.05$ & $P>0.05$ & $P>0.05$ & $P>0.05$ \\
\hline
\end{tabular}

\section{3 护理}

\section{1 光疗前护理}

光疗箱所处室内温度控制在 $23^{\circ} \mathrm{C} \sim 28^{\circ} \mathrm{C}$, 清洁、消毒蓝光 箱, 用箱前检查灯光是否全亮, 箱内湿化器加水 $2 / 3$ 满, 通电 后光疗箱内温度要调控在 $28^{\circ} \mathrm{C} \sim 32^{\circ} \mathrm{C}$, 箱内的湿度维持在 45\% 55\%, 将患儿手足戴好手、足套, 戴好眼罩, 穿好纸尿裤 后裸放人光疗箱, 头偏向一侧, 保持灯管与新生儿体表上下距 离均 $50 \mathrm{~cm}$ 。

\section{2 光疗过程中护理}

光疗过程中勤巡视，隔 $2 \mathrm{~h}$ 测量生命体征及翻身 1 次,及 时更换尿布并观察大便量及性状的变化, 注意观察有无发热、 皮疹、腹泻、脱水等副作用发生, 并注意观察有无病例发生, 以 便及时报告医生 ${ }^{[2]}$; 光疗过程中按需喂奶, 并采取侧卧位, 如有
患儿吃奶差, 应及时予以补液。

\section{3 光疗后护理}

解除患儿眼罩及手足套, 更换纸尿裤, 观察患儿皮肤有无 皮疹及破损,通过经皮测总胆红素值简单评价光疗效果; 做好 光疗期间所用物品的清洗、消毒工作。

\section{4 病情观察期间护理}

新生儿母乳摄入不足会使胎粪排出不畅, 导致未结合胆 红素增多使黄疸加重, 应密切观察患儿大便, 如有便秘及腹泻 应及时处理。

\section{4 讨论}

新生儿黄疸是新生儿阶段最常见的临床问题。黄疸表现 为未结合胆红素升高, 而未结合胆红素血症对中枢神经系统 有潜在毒性, 甚至可造成永久性后遗症, 应当予以积极治疗。

近年蓝光二极管 (LED) 作为一种新型光源。已被用于光 疗设备中, LED 冷光源蓝光有效光照强度为 $42 \mu \mathrm{W} / \mathrm{cm}^{2}$, 其光 谱波长范围 450 475nm。蓝光光源直接放在暖箱上进行光疗, LED 组治疗后 $24 、 48$ 小时经皮胆红素值明显下降, 且差异有 统计学意义。

LED 冷光源采用符合胆红素转化最佳吸收波长, 能够快 速、有效地使未结合胆红素的结构、性质和排泄途径发生改 变, 从而降低血清胆红素水平。LED 冷光源蓝光灯产生的辐射 热能极少, 不会增加体液丢失, 也不会影响喂养, 不产生紫外 线辐射能量,降低对婴儿皮肤伤害的风险, 可有效减少发热、 烦躁、呕吐、皮疹等光疗不良反应 ${ }^{[3]}$ 。

论文研究结果表明, LED 光疗优于普通光疗, 副作用少于 普通光疗, 故 LED 蓝光箱值得临床推广应用。

\section{参考文献}

[1]邵肖梅,叶鸿琩,丘小汕.实用新生儿学第四版[M].北京: 人民卫 生出版社,2011.

[2]李秋平,封志纯.美国儿科学会最新新生儿黄疸诊疗指南[J].继 续医学教育,2007(18):23-26.

[3]郭雯雯,胡子衡,孟娜娜, 等.加减保产无忧方预防新生儿黄疸的 临床应用研究[J].河北中医,2018,40(11):1656-1659. 